\title{
Fracture Behaviors of Graphene Sheets and Carbon Nanotubes
}

\author{
J. R. Xiao ${ }^{1^{*}}$ and J. W. Gillespie Jr. ${ }^{1,2,3^{* *}}$ \\ ${ }^{1}$ Center for Composite Materials, \\ 2Department of Materials Science and Engineering, \\ ${ }^{3}$ Department of Civil and Structural Engineering, \\ University of Delaware, \\ USA
}

\section{Introduction}

Graphene sheet is a one-atom-thick planar sheet of sp2-bonded carbon atoms that are densely packed in a hexagonal crystal lattice. Graphene is the basic structural element of some carbon allotropes including graphite, carbon nanotubes and fullerenes. Carbon nanotubes (CNTs) (Iijima, 1991) exhibit exceptional physical properties: small size, low density, high stiffness, high strength and excellent electronic and thermal properties (Dresselhaus et al., 1996; Wong et al., 1997; Che et al., 2000; Yakobson and Avouris, 2001; Thostenson et al., 2001). These exceptional mechanical and physical properties along with low weight of CNTs and recent improvements in their synthesis and purification techniques make CNTs excellent candidates for use in tailoring properties of composites (Wagner et al., 1998; Cadek et al., 2002; Dalton et al., 2003).

Molecular mechanics/dynamics and ab initio methods are suitable for studying the mechanical properties and fracture behaviors of grapheme sheet and nanotubes but are limited in scale and are computationally expensive. Recently, continuum mechanics based models for CNTs have been developed using the harmonic energy potential ( $\mathrm{Li}$ and Chou, 2003; Chang and Gao, 2003). These models reduce computational cost significantly, but can only be used to investigate elastic properties, such as the Young's modulus or Poisson's ratio. To fully predict the stress-strain relationship and failure mechanism of CNTs, these methods are not sufficient. The Brenner potential function (Brenner, 1990) is considered more accurate and versatile. It can handle changes in atom hybridization and bonds with atoms other than carbon. A continuum mechanics approach directly incorporating the Brenner potential function has been developed by Huang's group (Zhang et al., 2002; Jiang et al., 2003) to model elastic properties and stress-strain relationships of carbon nanotubes based on a modified Cauchy-Born rule. Based on the modified Morse potential function (Belytschko et al., 2001), which is simpler than the Brenner potential, the authors have developed models for perfect and defective CNTs (Xiao et al., 2005; Xiao et al., 2009). The developments have the ability to predict the ultimate stress and other mechanical properties, including nanotube's nonlinear stress-strain relationship. The analytical model (Xiao et al., 2005) has been extended to solve mechanical responses of defect-free single- and 
multi-walled CNTs under internal and external pressure loadings (Xiao et al., 2006; Xiao and Gillespie, 2006a) as well as aligned nanotube-composites (Xiao and Gillespie, 2006b). The analytical model not only provides simple closed-form solutions but also presents a better insight of the role of the atomic networks.

The present work discusses the atomistic based finite bond element model and its application to study the effects of Stone-Wales (5-7-7-5) defects on mechanical properties and fracture behaviors of graphene sheets and carbon nanotubes. The element formulation includes eight degrees of freedom reducing computational cost compared to the 12 degrees of freedom used in other FE type models. The coefficients of the elements are determined based on the analytical molecular structural mechanics model developed by the authors. The model uses the modified Morse potential to predict the Young's modulus and stressstrain relationship of perfect and defective nanotubes and graphene sheets. Research involving the Stone-Wales defect preformed by Tserpes, and Papanikos (2007) using the Finite Element (FE) based model does not include the deformation of the original nanotube structure around the nucleation site, which may not be true in general as atoms redistribute to minimize energy. The authors (Xiao et al., 2009) proposed a simple way to simulate the formation of a Stone-Wales (5-7-7-5) defect using an interaction mechanics method to calculate the deformations caused by the formation of a Stone-Wales.

The variation of ultimate stress, strain at failure, and Young's modulus values of carbon nanotubes and graphene sheets has been examined as a function of the distance between two defects aligned in the axial and hoop directions has been studied. The fracture failure patterns will be presented and discussed. The mechanical properties as a function of the number of defects in the hoop direction are also studied. It is found that the moduli are sensitive to the tube lengths when the total tube length is used to compute the overall effective axial strain. If one uses a characteristic length based on the size of the local perturbation in the deformation fieldcreated by the defect to define the strain, a size independent modulus can be obtained for the defective region. The diameter of the affected region $(2 \mathrm{~nm})$ from a single defect is defined as the defective length and is used for all different tube lengths examined in the present study. The effects of defect density on mechanical properties of tubes of any lengths are also discussed. Progressive failure in the post failure region is investigated and a few computational examples are discussed. The predictions compare favorably to the corresponding published results from experiments and numerical calculations (i.e tight binding or molecular dynamics) for armchair and zigzag carbon nanotubes.

\section{An atomistic based finite bond element model}

A single-walled carbon nanotube can be viewed as a hollow cylinder rolled from a graphene sheet, composed of carbon hexagons. The diameter of the nanotube can be calculated as $d=\sqrt{3\left(n_{1}^{2}+n_{2}^{2}+n_{1} n_{2}\right)} / \pi$, where $a=0.142 \mathrm{~nm}$ is the C-C bond length, and the pair of integers $\left(n_{1}, n_{2}\right)$ are indices to represent its helicity such as armchair $\left(n_{1}=n_{2}\right)$ and zigzag $\left(n_{2}\right.$ $=0)$ nanotubes.

There are several different potential functions available (Brenner, 1990; Abell, 1985; Tersoff, 1988) for describing C-C bond interaction other than simple harmonic functions. Among them, the modified Morse potential function (Belytschko et al., 2001) is simple and is used in the present study. The modified Morse potential function was correlated to the Brenner potential function for strains below $10 \%$. In this paper, a new term $E_{\text {torsion }}$ is added to 
consider the bond energy due to angle variation of bond twisting $\Delta \phi$ which has been found to be negligible for defect-free tubes ( $\mathrm{Li}$ and Chou, 2003) and was neglected in earlier studies (Chang and Gao, 2003; Xiao et al., 2005; Xiao et al., 2006; Xiao and Gillespie, 2006a; Xiao and Gillespie, 2006b). However, this term may play a more important role in defective tubes. The energy potential function is given as follow:

$$
E=E_{\text {stretch }}+E_{\text {angle }}+E_{\text {torsion }}
$$

where $E_{\text {stretch }}$ is the bond energy due to bond stretch $\Delta r$, and $E_{\text {angle }}$ is the bond energy due to bond angle variation $\Delta \theta$, and

$$
E_{\text {torsion }}=\frac{1}{2} k_{\phi}(\Delta \phi)^{2}
$$

The parameters associated with the terms $E_{\text {stretch }}$ and $E_{\text {angle }}$ can be seen in Ref (Belytschko et al., 2001). The force constant associated with the term (2) is taken as ( $\mathrm{Li}$ and Chou, 2003; Cornell et al., 1995; Jorgensen and Severance, 1990) $k_{\phi}=0.278 n N-n m / \mathrm{rad}^{2}$.

The stretch force, the angle-variation moment and the torsional moment can be obtained from differentiations of Eq. (1) as functions of bond stretch, bond angle variation and torsion angle variation, respectively:

$$
\begin{gathered}
F(\Delta r)=2 \beta D_{e}\left(1-e^{-\beta \Delta r}\right) e^{-\beta \Delta r} \\
M(\Delta \theta)=k_{\theta} \Delta \theta\left[1+3 k_{\text {sextic }}(\Delta \theta)^{4}\right] \\
M_{\phi}(\Delta \phi)=k_{\phi} \Delta \phi
\end{gathered}
$$

A constant bond torsional stiffness is implied by Eq. (3c). The stretch stiffness and the anglevariation stiffness can be further obtained from differentiations of Eqs. (3a-3b) as functions of bond stretch and bond angle variation, respectively:

$$
\begin{aligned}
& k_{r}(\Delta r)=2 \beta D_{e}^{2}\left(1-2 e^{-\beta \Delta r}\right) e^{-\beta \Delta r} \\
& k_{\theta}(\Delta \theta)=k_{\theta}\left[1+15 k_{\text {sextic }}(\Delta \theta)^{4}\right]
\end{aligned}
$$

Analytical solutions for predicting nonlinear mechanical behaviors of defect-free SWCNTs have been investigated by using an effective "stick-spiral" model based on a unit cell approach (Xiao et al., 2005). The effective "stick-spiral" model uses a stick with Eq. (3a) to model the force-stretch relationship of the C-C bond and a spiral spring with Eq. ( $3 b)$ to model the angle bending moment resulting from an angular variation of bond angle. The stick is assumed to have an infinite bending stiffness and finite torsional stiffness. One can include the torsional term by using the stick with Eq. (3c).

For a defective nanotube with loss of local symmetry, the unit cell approach becomes inappropriate, and a generalized molecular mechanics (MM) model (Sears and Batra, 2004) or a finite element (FE) type model ( $\mathrm{Li}$ and Chou, 2003) are needed to include the entire molecular structure system of the defective nanotube in order to consider the effect of 
defects on its mechanical response. In this paper, we propose a new FE type model for nanotubes based on the effective "stick-spiral" model. Bond elements are developed for simulating deformation modes of the chemical bonds.

Typically the FE type model ( $\mathrm{Li}$ and Chou, 2003) uses a beam element (Kanchi 1993) with sectional stretch stiffness for the force-stretch relationship of the C-C bond, sectional flexural rigidity for the angle bending moment (Eq. 3b), and sectional torsional stiffness for the torsional moment (Eq. 3c). Each element has 12 degrees of freedom. The major differences between the FE type model and the effective "stick-spiral" model are the assumptions made with respect to the bending stiffness (flexible vs rigid). The bond element used in the present paper has infinite bending stiffness (the stick) with finite bending stiffness of the two end joints (the spirals) indicated by the square box shown in Fig. 1. Each of the three molecular deformation modes (stretching, angle variation, and angle torsion) are represented by tension, bending and torsion of a bond element with 8 degrees of freedom $u_{e}=\left[\begin{array}{llllllll}u_{x i} & u_{y i} & u_{z i} & \phi_{i} & u_{x j} & u_{y j} & u_{z j} & \phi_{j}\end{array}\right]$. The element can be stretched (pure tension) and torqued (pure torsion) along its axial direction and bent by relative transverse displacement without angle changes at the two ends. The bond angle variation of the bond element can be associated with the relative transverse displacement $(e)$ between the two ends as $d \theta=e / a$ (Fig. 1c). The present approach, designated the finite bond element model, is expected to give the same solution as the "stick-spiral" model (Xiao et al., 2005; Chang and Gao, 2003) for defect-free CNTs. The stick-spiral model with infinite bending stiffness represents the true physical deformation modes and is able to predict both in-plane stiffness (Young' modulus) and Poisson's ratio of CNTs accurately.

For the bond element defined in Fig. 1 in a three-dimensional space, the elemental equilibrium equation can be established for every bond element. The final system of equations with appropriate boundary conditions imposed can be solved by the displacement-control Newton-Raphson method. A MATLAB program has been written based on finite bond element method for SWCNTs subjected to tensile loadings.

\section{Validation on defect-free SWCNTs}

The calculations based on our FE-based finite bond element model will be validated by comparing with the results obtained from the analytical model (Xiao et al., 2005) for defectfree CNTs. The initial equilibrium state of the atomistic system of a CNT is created based on the $a b$ initio calculations (Ye et al., 2001) where the approximate expressions of angle $\alpha$ and $\beta$ of armchair nanotubes are taken as $\alpha \approx 2 \pi / 3$ and $\beta=\pi-\operatorname{arcos}\left[0.5 \cos \left(\pi / 2 n_{1}\right)\right]$, respectively. The angle $\alpha$ and $\beta$ for zigzag nanotubes as $\alpha \approx 2 \pi / 3$ and $\beta=\operatorname{arcos}\left[0.25-0.75 \cos \left(\pi / n_{1}\right)\right]$. The displacement boundary conditions for the simulations are shown in Figure 2 . The atoms on the bottom edge of the tube are fixed in the axial (z) direction. One atom is fully fixed in all three $(x-, y-$ and $z)$ directions to prevent rigid body rotations. The other end of the CNT was axially displaced incrementally to introduce load into the tube.

The interatomic force per atom $(f)$ is calculated for all atoms along the end of the nanotube where the displacement is prescribed. For defect-free nanotubes, the force is identical for all atoms on the end. The axial strain of the CNT is computed as $\varepsilon=\Delta L / L_{0}$, where $L_{0}$ is the initial length of the CNT. The force-strain relationship of nanotubes is predicted using the procedures outlined above. Fig. 3 shows the computed force-strain relationships for 


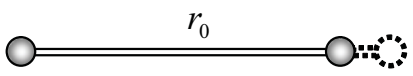

(a) Stretching

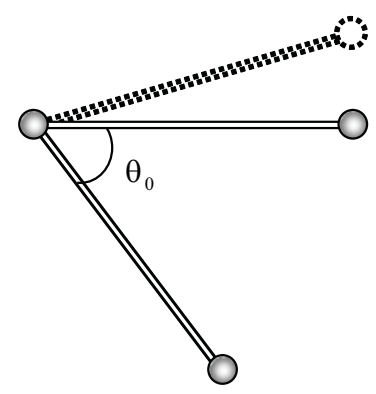

(b) Angle variation

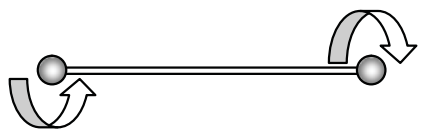

(c) Torsion

MM Modes

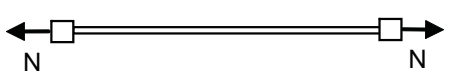

(d) Tension

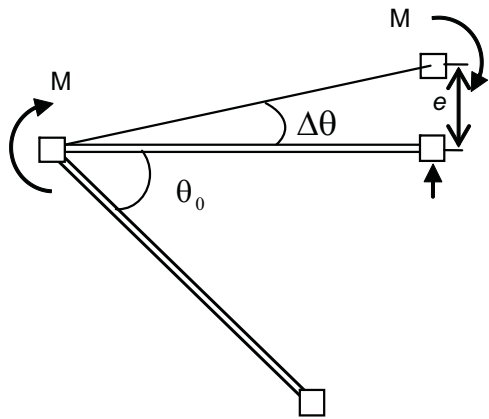

(e) Bending with relative transverse displacement

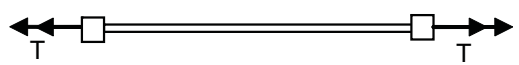

(f) Torsion

Fig. 1. Molecular mechanics modes are compared with finite bond element method

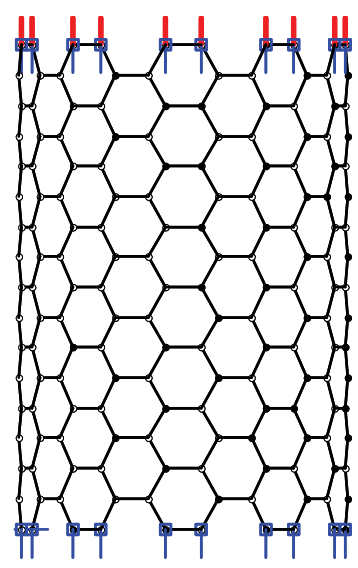

a $(10,10)$ nanotube

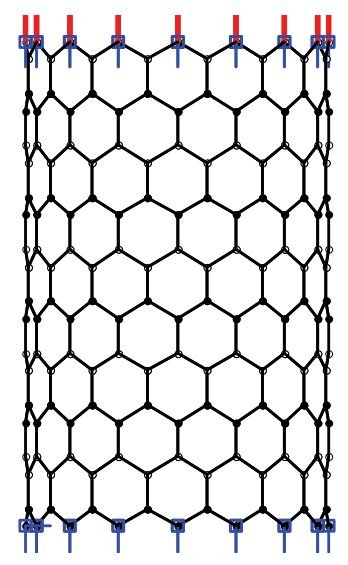

a $(16,0)$ nanotube

Fig. 2. Atomistic armchair and zigzag nanotubes (blue bar denotes constrained displacement and red bar indicates prescribed displacement) 
armchair and zigzag nanotubes. Only two different types of nanotubes (i.e. a $(12,12)$ armchair and a (20,0) zigzag) are presented for illustration purpose.

The numerical procedure is able to give the post failure behavior (i.e. beyond the inflection point where the maximum of the interatomic force occurs) as shown in Fig. 3. Similar predictions have been reported in the study (Jiang et al., 2003). However, the accuracy of the predicted post failure response (dash lines) is limited to the strains shown in Fig. 3 (vertical solid line) since the simple interatomic potential function used is not capable of describing the behavior of the nanotube after the bonds are broken where the formation of new bonds, rehybridization and structural transformations may occur.

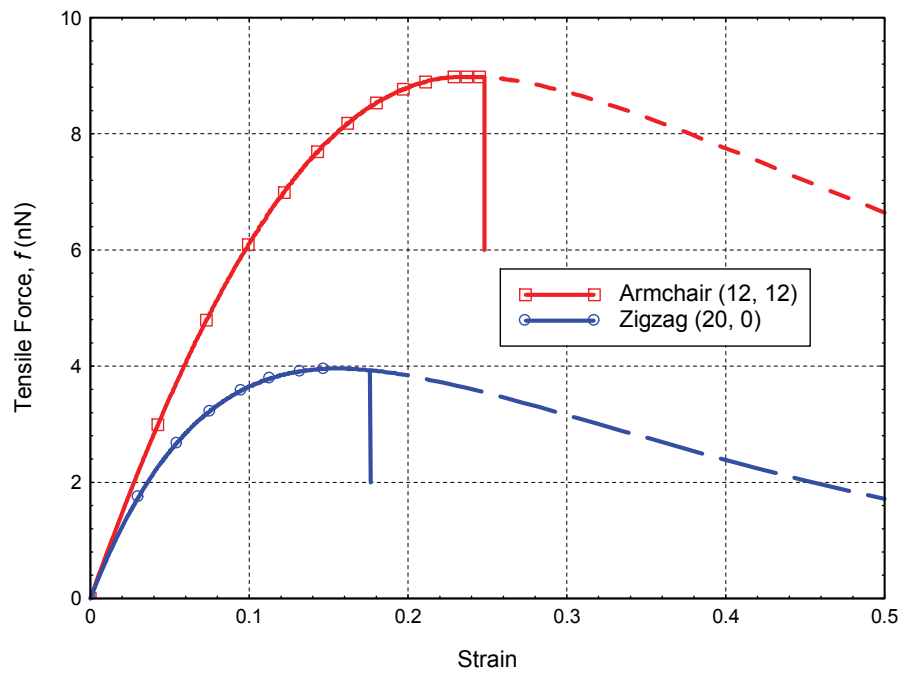

Fig. 3. Tensile force-strain curves for armchair and zigzag nanotubes

From the experimental (Yu et al., 2000) and theoretical (Belytschko et al., 2001) studies on the tensile behavior of nanotubes, it was found that the stress exhibits a sudden drop to zero when stress reaches the tensile strength and the fracture is brittle. The predicted maximum load corresponding to the inflection point is taken as the tensile capacity of the defect free nanotube in the present study which is highlighted in Fig. 3.

It should be noted that the present method does not require a tube thickness to be defined. However, in order to compare the results with published data, conventional moduli and strengths can be calculated using an assumed wall thickness for CNTs (e.g. $t=0.34 \mathrm{~nm}$ is used in the literature). The total force carried by the CNTs can be given as $F=2 n_{1} f$, and then the stress can be computed as $\sigma=F / \pi d t$ and the Young's modulus as $E=\frac{F / \pi d t}{\Delta L / L_{0}}$, respectively. It is not surprising that the predicted tensile behaviors are almost identical to those of the analytical molecular mechanics model (Xiao et al., 2005) because the coefficients used in the present atomistic based FE model are the same as those used in the analytical model. The negligible difference (less than $0.5 \%$ ) is attributed partially to the effects of the torsional mode and numerical round-off error. Consequently, the present study also confirmed that the torsional term in the potential energy is negligible when the defect free tubes are subjected to tensile loading which justifies the assumptions made in other studies. 
The size-dependent feature of in-plane stiffness and Poisson's ratio can also be captured by the present model. The Young's moduli for both armchair and zigzag nanotubes decrease with decreasing tube diameter and approach the predicted graphite value $\left(383 \mathrm{~J} / \mathrm{m}^{2}\right.$, corresponding to a Young's modulus of $1.13 \mathrm{TPa}$ with thickness of $0.34 \mathrm{~nm}$ ) when the tube diameter is increased. The Poisson's ratios for both armchair and zigzag tubes decrease with increasing tube diameter and approach the limit value of 0.20 for graphene sheet, which agrees with both molecular dynamics simulations and experimental values. It should be noted that the similar FE based model ( $\mathrm{Li}$ and Chou 2003) predicted a very low value $(<0.08)$ for the Poisson's ratios of graphene sheets and nanotubes. Consequently, the present formulation overcomes this limitation in previous work. In the context of the present study, accurate modeling of Poisson interactions in the prediction of defect formation is important as shown in the next section.

The predicted nonlinear behaviors of nanotubes are very similar to results calculated from molecular dynamics (Belytschko et al., 2001). The predicted strengths and failure strains are significantly higher than the experimental values $(11 \sim 63 \mathrm{GPa}$ for strength and $10 \% \sim 13 \%$ for failure strain) (Yu et al., 2000). This difference can be partially explained by the presence of defects that reduce stiffness and strength. The mechanical behavior of defective nanotubes is studied in the following section.

\section{Progressive failure of defective SWCNTs}

\subsection{Stone-wales defect formation}

Various types of defects exist in CNTs, such as vacancies and topological defects (5-7-7-5). The Stone-Wales 5-7-7-5 defect involves the 90 rotation of a carbon bond with a new configuration as shown in Fig. 4. The effect of the SW defect on the configuration is believed to be local and limited to atoms in the neighborhood of the defect. Atoms far away from the defect undergo uniform deformation and their geometry configurations are the same as the defect-free tubes. For atoms near the defect, an interaction (similar to contact) mechanics concept is used to determine their initial equilibrium positions, which is possible by treating the defect formation as the result of interaction between two deformable 'bodies' as shown in Fig. 5. 'Inserting' the body $b$ of a zigzag unit into the body $a$ with an armchair hole as

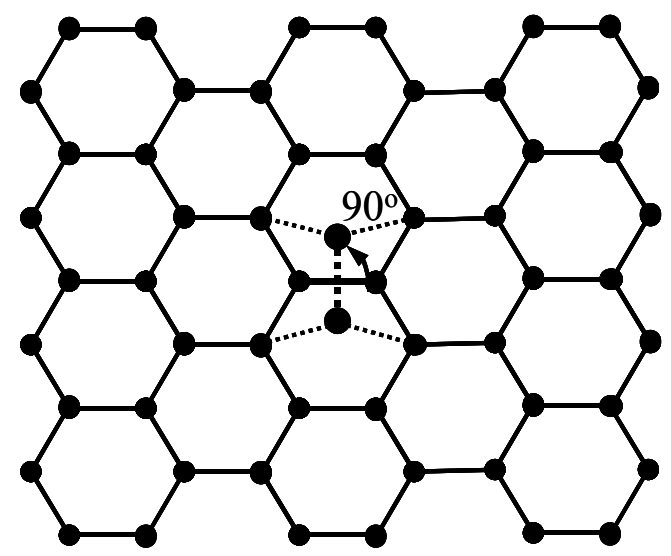

Fig. 4. SW defect generated by rotating the $\mathrm{C}-\mathrm{C}$ bond 
defined in Fig. 5 and forcing them to be coincident at four specific locations will cause reconfiguration of the system within a localized region because of the geometrical distortions that exist at the four locations. Assuming the same in-plane properties for each body (governed by the same potential function), self-equilibrating 'residual' or internal forces will be generated between disturbed atoms if we assume that there is no net atomic force between atoms at equilibrium state of defect-free region (reference state). The resulting configuration corresponds to the minimized energy state of a defective graphene sheet and CNT.

The finite bond element method is used for describing atomistic force-displacement interaction for both bodies to study how the two regions conform to each other. The two deformable bodies $\Omega^{e}(e=a, b)$ are shown in Fig. 5 . The interaction sites (contact 'surface') consists of four atoms (1, 2, 3 and 4) that exist in both bodies with prescribed displacements along $\Gamma_{u}^{e}$ and tractions applied on $\Gamma_{t}^{e}(e=a, b)$. If the initial gap along the interaction surface of the two bodies is denoted by $g\left(=\left(u_{x i} u_{y i} u_{z i}\right), i=1,2,3,4\right)$ the interaction condition can be described in the form of inequalities as:

$$
\begin{aligned}
& F_{c} \leq 0 \quad g \geq 0 \\
& g F_{c}=0
\end{aligned}
$$

where $F_{c}\left(\begin{array}{lll}F_{x i} & F_{y i} & F_{z i}\end{array}\right)$ is the interaction force vector of the four interfacial atoms. For each body, displacement and force fields are determined by the molecular mechanics and can be written in the following condensed matrix form:

$$
\left[\begin{array}{cc}
K_{a} & 0 \\
0 & K_{b}
\end{array}\right]\left\{\begin{array}{l}
u_{a} \\
u_{b}
\end{array}\right\}=\left\{\begin{array}{c}
F_{t}-F_{c} \\
F_{c}
\end{array}\right\}
$$

in which $u_{a}$ and $u_{b}$ are components of the displacement vector (same size as the gap vector $g$ ) of the four atoms in the body $a$ and $b$, respectively. $F_{t}$ is the applied external force vector. $K_{a}$ is the condensed stiffness matrix of the body $a$ with the displacements $u_{a}$ as the basic unknowns and contents of stiffness contributions from all elements of the body $a . K_{b}$ is the condensed stiffness matrix of the body $b$. By introducing the following transformation:

$$
\left\{\begin{array}{l}
u_{a} \\
u_{b}
\end{array}\right\}=\left[\begin{array}{ll}
I & I \\
0 & I
\end{array}\right]\left\{\begin{array}{c}
u_{a} \\
g
\end{array}\right\}
$$

where $[I]$ is the identity matrix. Eq. (9) can be easily transformed to:

in which

$$
K g=F+F_{c}
$$

$$
\begin{gathered}
K=K_{a}-K_{a}\left[K_{a}+K_{b}\right]^{-1} K_{a} \\
F=F_{t}-K_{a}\left[K_{a}+K_{b}\right]^{-1} F_{t}
\end{gathered}
$$

So that the force $F_{c}$, which is required to close the gap to achieve coincident atom locations, can be calculated from Eq. (11) for the initial $(F=0)$ and/or loaded states as: 


$$
F_{c}=-K g-F
$$

The gap function is generated by aligning nodes 1 and $1^{\prime}$ of two bodies in Fig. 5 . The boundary condition applied to the system matrix in Eqs. 11 and 12 is that node 1 is fixed. With the force $F_{c}$ known one can determine the equilibrium positions of atoms in the defective system which corresponds to the minimized system energy. It should be noted that the proposed analysis involves a nonlinear procedure since the modified Morse potential energy function is used. The formation energy can be calculated as $\int F_{c} d g \approx \sum_{i} 0.5 F_{c}^{i}\left(d g^{i}+d g^{i+1}\right)$ or simply calculated as $0.5 F_{c} \mathrm{~g}$ without significant loss of accuracy.

The formation energy of a single SW defect occurring at the center of nanotubes is slightly different for various tube diameters examined. A typical value of $2.86 \mathrm{eV}$ for a $(12,12)$ tube agrees with the range of published values (2.5 3.5eV) (Nardelli et al., 1998; Pan et al., 2000). Generally the larger diameter requires the higher formation energy with a plateau value of $2.97 \mathrm{eV}$ for a graphene sheet based on the present model as shown in Fig. 6. The calculation of the defect formation energy is more complicated than the approximate method presented in this work. However, we have compared our simple approach to predict the defect formation energy and found reasonable accuracy with published molecular dynamic simulations. The formation of SW defect causes elongation of the local tube structure along the axial direction, but also shrinking along the hoop direction. Fig. 7 shows the affected area and configuration due to the formation of the SW defect in a graphene sheet and the $(12,12)$ CNT. The shrinking can be seen from the side view of the defective tube as shown in Fig. 7.

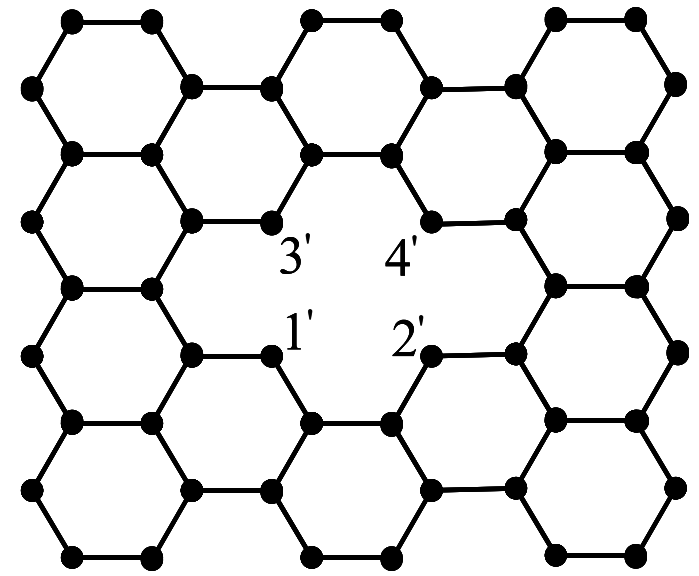

(a) Ideal armchair configuration with a hole

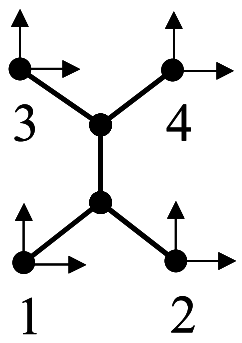

(b) A zigzag unit

Fig. 5. Modeling of atom position rearrangements due to SW defect formation

Based on our simulations, the affected region in a tube/sheet is localized and covers four neighboring rings (hexagons) around the defect. The affected area is slightly sensitive to the tube diameter when the tube diameter is smaller than $2 \mathrm{~nm}$ and becomes insensitive for larger diameters. The molecular structure outside the region remains undisturbed. All chemical bonds within the affected region are preloaded by the presence of the SW defect. 
With the application of external tensile force on the tube ends, the defect region becomes the weakest point and will serve as a damage nucleation site for progressive failure as given in the following section.

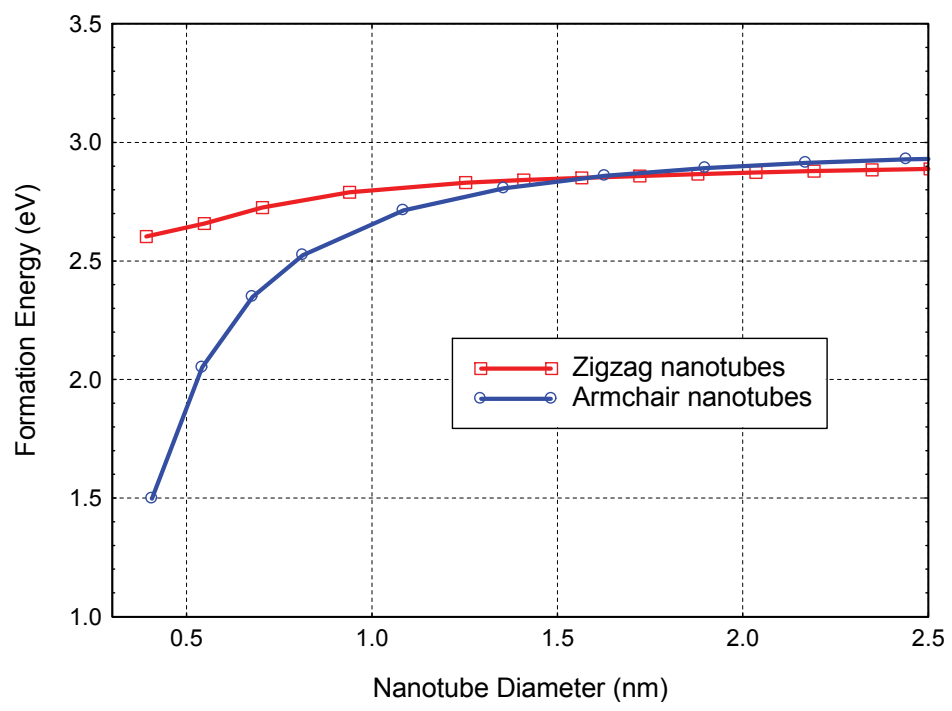

Fig. 6. Formation energy of SW defect vs nanotube diameter

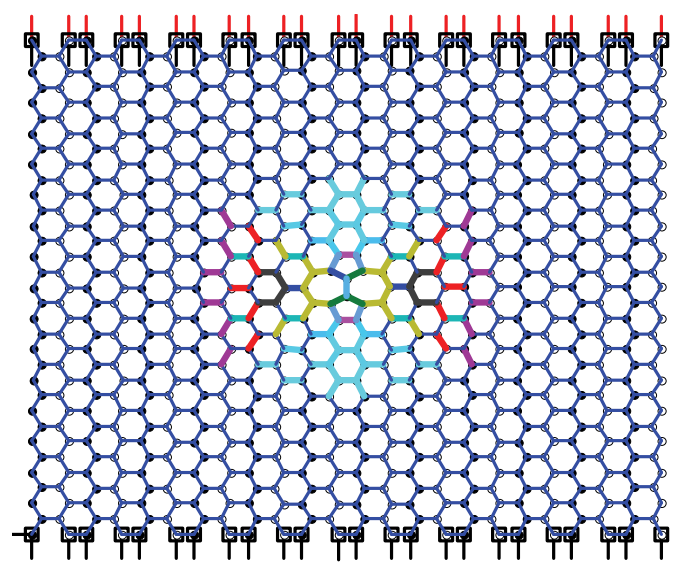

Graphene sheet

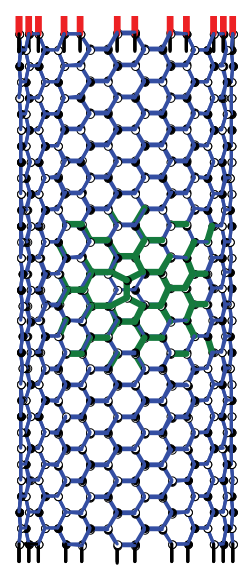

Front view

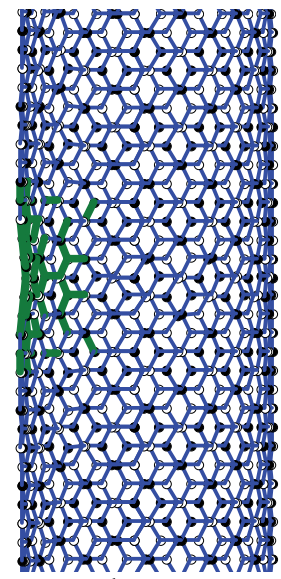

Side view

Fig. 7. Formation of SW defect in a graphene sheet and a $(12,12)$ SWCNT

\subsection{Progressive failure analysis}

After the defect formation in a graphene sheet or nanotube, one can apply further load (tension) onto the defective sheet and tube as shown in Fig. 7, where the load and boundary conditions are applied in the same fashion as described above. The tube length is taken as 6 $\mathrm{nm}$ for all simulations. 
The bond breaking criterion brings an important issue in simulating fracture of solids. Very often, a cut-off distance $r_{c f}$ is used in atomistic simulations. Such distance-based criterion is adopted in the present study. In our analytical study (Xiao et al., 2005), the inflection point (corresponding to a $r_{c f}=0.168 \mathrm{~nm}$ ) was used. In the present study the effect of the cut-off distances on the fracture and failure of defective graphene sheets and nanotubes will be investigated.

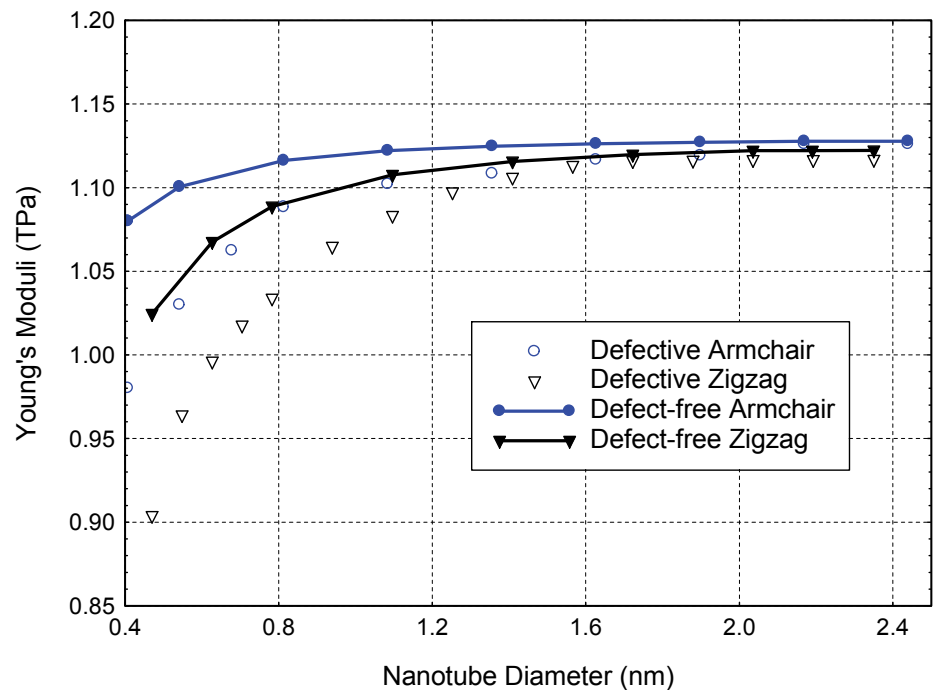

Fig. 8. Young's moduli of carbon nanotubes vs tube diameter

Different cut-off distances have been suggested (Xiao et al., 2005; Lu and Bhattacharya, 2005; Huhtala et al., 2004; Xia et al., 2002; Dumitrica et al., 2003). It has been found that there is no significant change in the critical bond-breaking force with values ranging from $0.17 \mathrm{~nm}$ to 0.19 $\mathrm{nm}$. Clearly, the cut-off distance used in our analytical study (Xiao et al., 2005) is close to this range. A value of $r_{c f}=0.175 \mathrm{~nm}$ is adopted in the present study unless otherwise stated. As shown in the study (Belytschko, et al., 2003), the force field shape of the modified Morse potential function is essentially the same as that of the Brenner potential function before the inflection point (i.e. the maximum of the interatomic force) and totally different after the inflection point. Belytschko, et al. (2003) reported that the fracture is essentially independent of the separation energy and depends primarily on the inflection point of the interatomic potential, i.e. the shape of the potential function after the inflection point is not important to fracture behavior. Consequently, any cut-off distances beyond the inflection point will not affect the fracture response but will significantly increase the computational cost.

Fig. 8 shows the calculated Young's modulus of defective nanotubes (open points) compared with defect-free tubes (solid lines). It can be seen that the Young's moduli are size dependent for both defective and defect-free tubes, and the Young's moduli of defective tubes are reduced because of the presence of SW defect, particularly for small diameter tubes $(10 \%$ reduction for diameters ranging from $0.4-0.6 \mathrm{~nm})$. Such reduction becomes insignificant for larger diameter $(>2 \mathrm{~nm})$ tubes simply because the ratio of the affected region (almost constant) over the whole area along the hoop direction decreases as the tube diameter increases. 


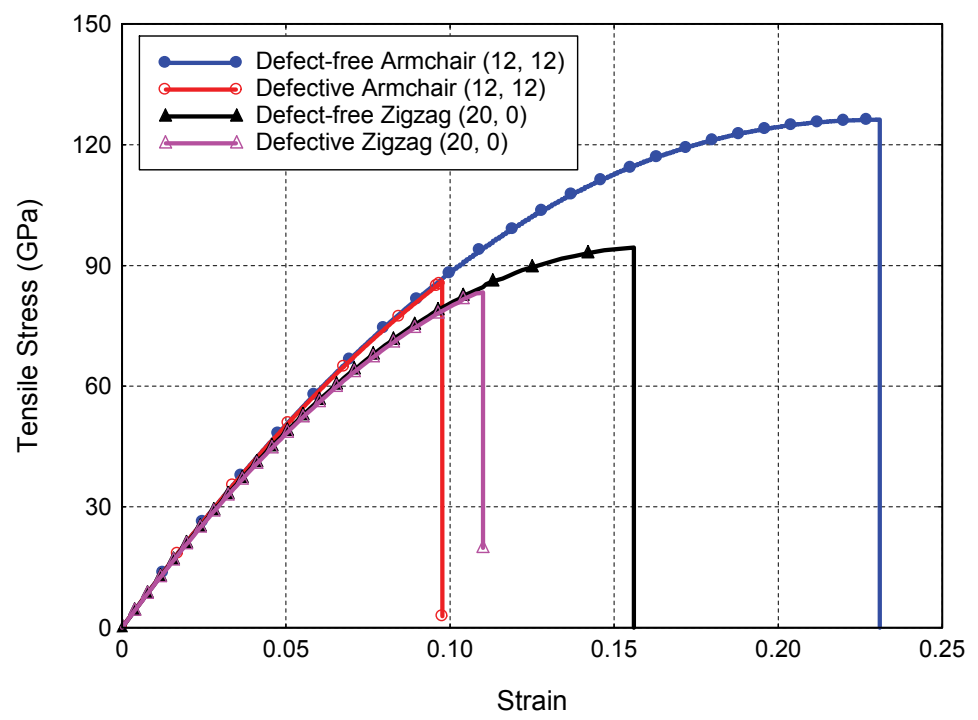

Fig. 9. Tensile force-strain curves of carbon nanotubes

Fig. 9 shows the calculated stress-strain relationships for armchair and zigzag nanotubes with and without 5-7-7-5 (SW) defect at the center. Again, only two different types of nanotubes $((12,12)$ armchair and $(20,0)$ zigzag with diameter around $1.6 \mathrm{~nm})$ are presented. As can be seen from Fig. 9, the predicted tensile strength $(85.9 \mathrm{GPa})$ of defective armchair nanotubes is much less than that $(126.2 \mathrm{GPa})$ of defect-free armchair nanotubes (reduced $32 \%$ ). Whereas, the effect of the SW defect on the failure strength of zigzag tubes is less significant for armchair tubes because of nanotube chirality. The failure strength of defective $(20,0)$ zigzag tube is $83.3 \mathrm{GPa}$, which is $12 \%$ reduced from the pristine value of $94.5 \mathrm{GPa}$. The predicted failure strains in the present study are $9.8 \%$ for the defective $(12,12)$ armchair nanotube $(23.1 \%$ for the defect-free), and $11.0 \%$ for the defective $(20,0)$ zigzag nanotube (15.6\% for the defect-free). All these predictions agree well with the MD results (Belytschko, et al., 2003). It should be noted that the predicted strengths are still significantly higher than the experimental values (11 $\sim 63 \mathrm{GPa})$ of Yu et al. (2000) though the predicted failure strains are comparable to the measured results $(10 \% \sim 13 \%)$ (Yu et al., 2000). This issue can be partially explained by the present of multiple SW defects (Lu, B. Bhattacharya, 2005; Dumitrica et al., 2003) as well as other types of defects (Xiao and Hou, 2006).

Tubes are found to exhibit brittle behavior at fracture. Once the tube deformation reaches a critical level (corresponding to the bond cutoff distance), atomic bonds break successively and lead to a complete fracture with little strain applied. The effect of the cutoff distance on the progressive failure has been examined with three different cutoff values $(0.168,0.175$ and $0.185 \mathrm{~nm}$ ) used. It is found that the ultimate strengths and failure strains are almost identical (difference is less than $0.1 \%$ ) for all three values examined and the failure is brittle no matter what value is used for the cutoff distance. However, the computing time with $r_{c f}$ $=0.185 \mathrm{~nm}$ is much longer than those with other two smaller values. The effect of the torsional term on the mechanical behaviors of defective tubes was also assessed. The present study shows that the torsional term in the potential energy still plays a minor role when the defective tubes are subjected to tensile loading. 


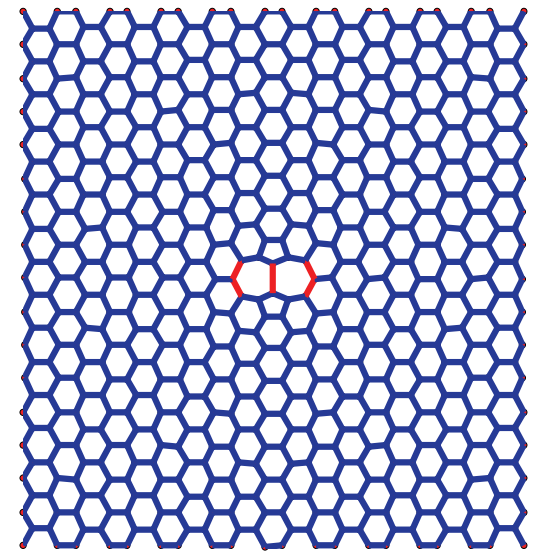

$9.69 \%$

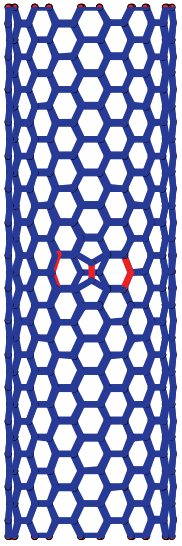

$9.23 \%$
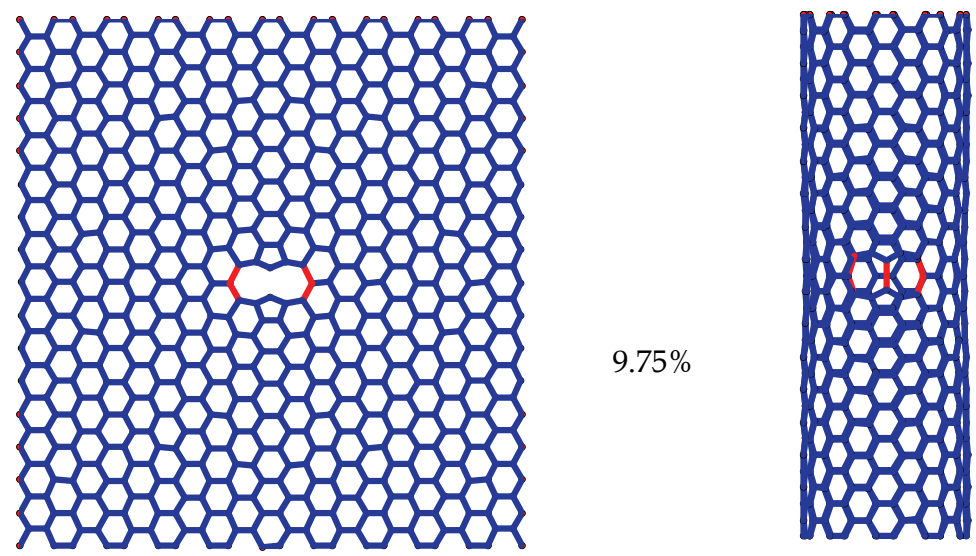

$9.35 \%$
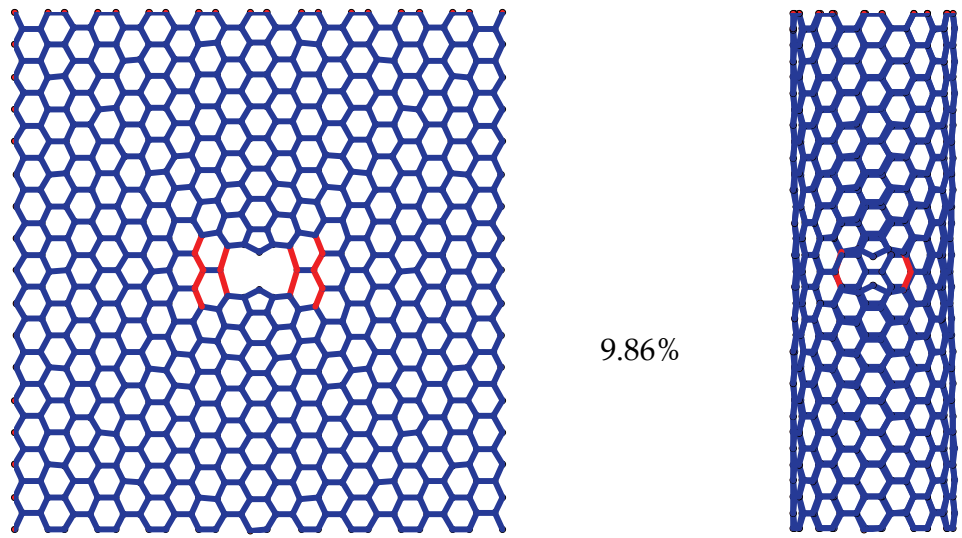

$9.45 \%$

$9.86 \%$ 

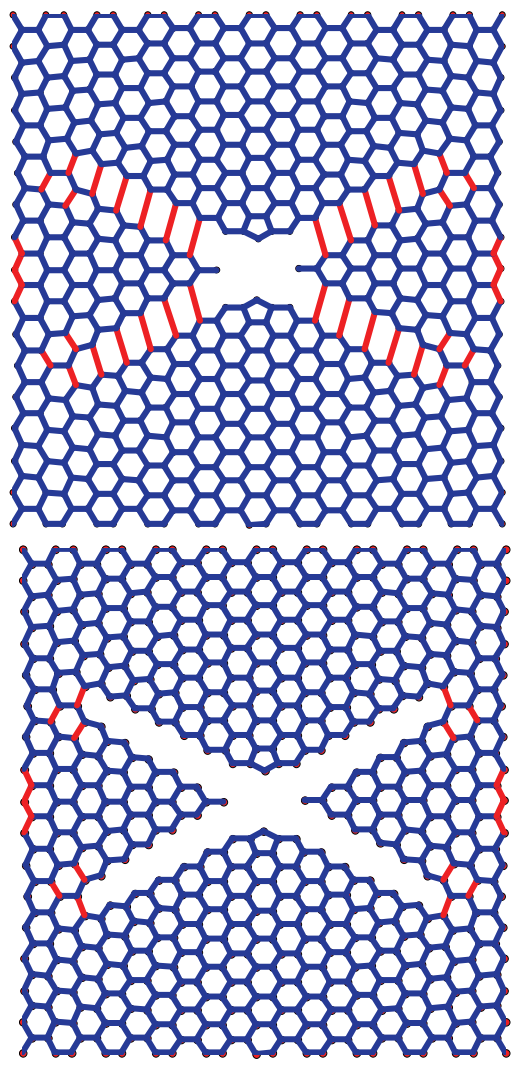

(a) a grahpene sheet

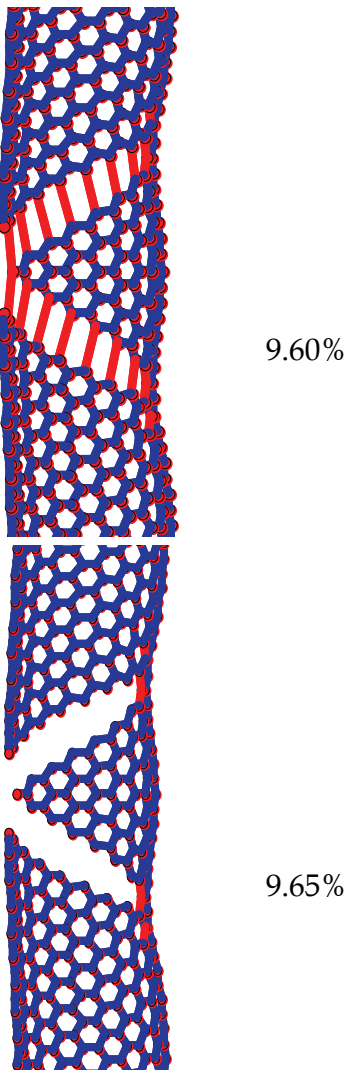

(b) a $(10,10)$ armchair nanotube

Fig. 10. Fractured profiles at different strains of a defective graphene sheet and a defective nanotube under tension

Fig. 10 shows the crack evolution (elimination of failed bond elements based on the cutoff distance failure criterion) at different strain levels for a graphene sheet and an $(10,10)$ armchair CNT. Similar failure patterns can be seen between the graphene sheet and CNT which exhibits diagonal crack paths. The failure pattern in the CNT wraps around the tube in the $\pi / 4$ direction along its circumference, which is similar to MD simulations (Belytschko, et al., 2003; Lu and Bhattacharya, 2005).

\subsection{Effects of multiple stone-wales defects}

The present study also considers CNTs with multiple defects along its axial direction and hoop direction, respectively. The effect of the distance between adjacent defects and the number of defects on the mechanical behaviors of defective graphene sheets and CNTs will be examined. The developed MATLAB program of the finite bond element model has been further improved to be able to form several Stone-Wales defects randomly at multiple points in the CNT structure. The present method for creating multiple defects on a nanotube is similar to creating a single defect. Once the first defect is created at a given location, any 
deformations of the tube are taken into account for the next defect. The defects are therefore created at specific locations sequentially, not simultaneously. The effect of randomly distributed multiple defects on mechanical properties of nanotubes has been studied by $\mathrm{Lu}$ and Bhattacharya [17] using MD. Predictions on mechanical properties of CNTs with multiple defects based on continuum mechanics based models have not been well studied. The present study provides understanding of the effects of multiple defects on the Young's modulus, ultimate strength, and strain at failure of the defective CNTs. After defect formations in a graphene sheet or nanotube, one can apply further load (tension) onto the defective sheet and tube as shown in Fig. 11, where the load and boundary conditions are applied in the same fashion as described above. Different tube lengths are examined.
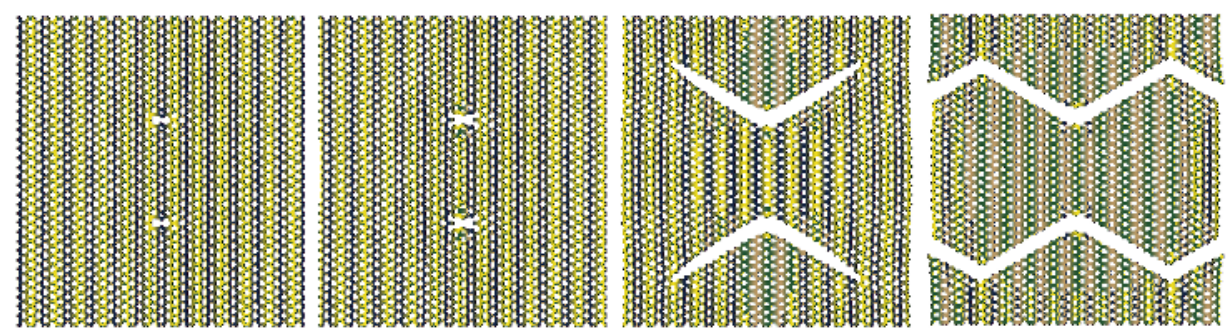

(a) Graphene sheet
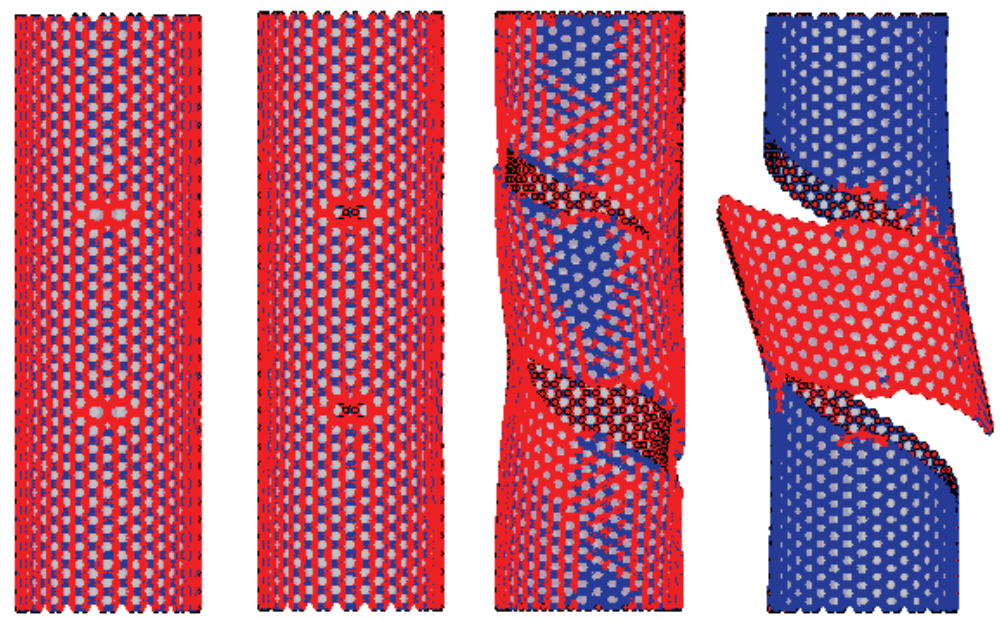

(b) CNT

Fig. 11. Fracture pattern of a graphene sheet and tube with two defects along its axial direction

\subsubsection{Effects on young's modulus}

From our simulations, it has been found that graphene sheets and CNTs with multiple defects along its axial direction (with separation distance larger than 2nm) show similar moduli and strengths as those with single defect, because the determining factor is the weakest cross-section. It should be noted that there is no defect interactions when the separation distance is greater than $2 \mathrm{~nm}$. The present study focuses on the effects of two 
defects located at the same axial location but separated by a prescribed angle around the circumference. We look at a $(20,20)$ CNT with a diameter of $2.71 \mathrm{~nm}$. Based on our previous study (Xiao et al., 2005), the Young's moduli of nanotubes with a diameter larger than 2nm are insensitive to their diameters. Fig. 12 compares the calculated Young's Moduli of the CNT with different angles (in degree) between the two defects for 4 different tube lengths. The effect that the difference in angle between defects has on the modulus is negligible for angles $>70$ degree (around $2 \mathrm{~nm}$ separation). Similar studies carried out on $(10,10),(17,0)$, and $(35,0)$ tubes of varying lengths yield similar results. It is also noticed that the shorter the tube length is, the lower Young's Modulus, because the ratio of the affected area over the total length is larger for a short tube length. This is a consequence of how axial strain of the tube is defined. In our study we have adopted the definition commonly used in experimental studies.

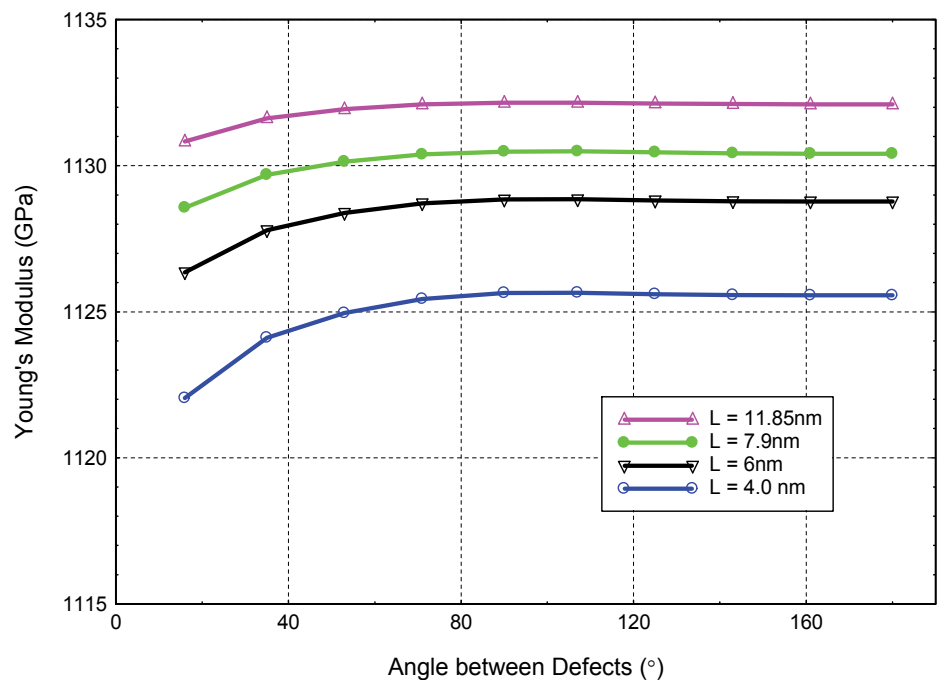

Fig. 12. Young's modulus vs. angle between two defects for $(20,20)$ CNTs with different lengths (Young's modulus calculated using total tube length)

To better understand these local effects on modulus one can separate the defective tubes into two different regions, i.e. the affected region which is about $2 \mathrm{~nm}$ long (correlates to the failure pattern of a single defect as shown in Xiao, et al., (2009), and unaffected region (total length minus $2 \mathrm{~nm}$ ). In the unaffected region the Young's modulus is the same as the defectfree tubes reported previously (Xiao et al., 2005). In the affected region, local strains are defined as $\varepsilon=\Delta L_{D} / L_{D 0}$, where $L_{D 0}$ is the initial length of the affected region (i.e. $2 \mathrm{~nm}$ ) and $\Delta L_{D}$ is the change in length of the affected region. Consequently, two different moduli can be defined, i.e. (a) apparent modulus $E_{\text {total }}$ based on the total length as shown in Fig. 12, and (b) defective modulus $E_{\text {local }}$ based on the local affected length as shown in Fig. 13.

In Fig. 12, a plateau modulus is reached for separation angles greater than 72 degrees for all tube lengths. The magnitude of this plateau modulus increases as the tube length increases. For infinitely long tubes, the plateau modulus approaches the modulus of the defect free tube. For angles less than 72 degrees, modulus decreases from the associated plateau level due to defect interactions (i.e. distance between defects is less than $2 \mathrm{~nm}$ affected zone). 


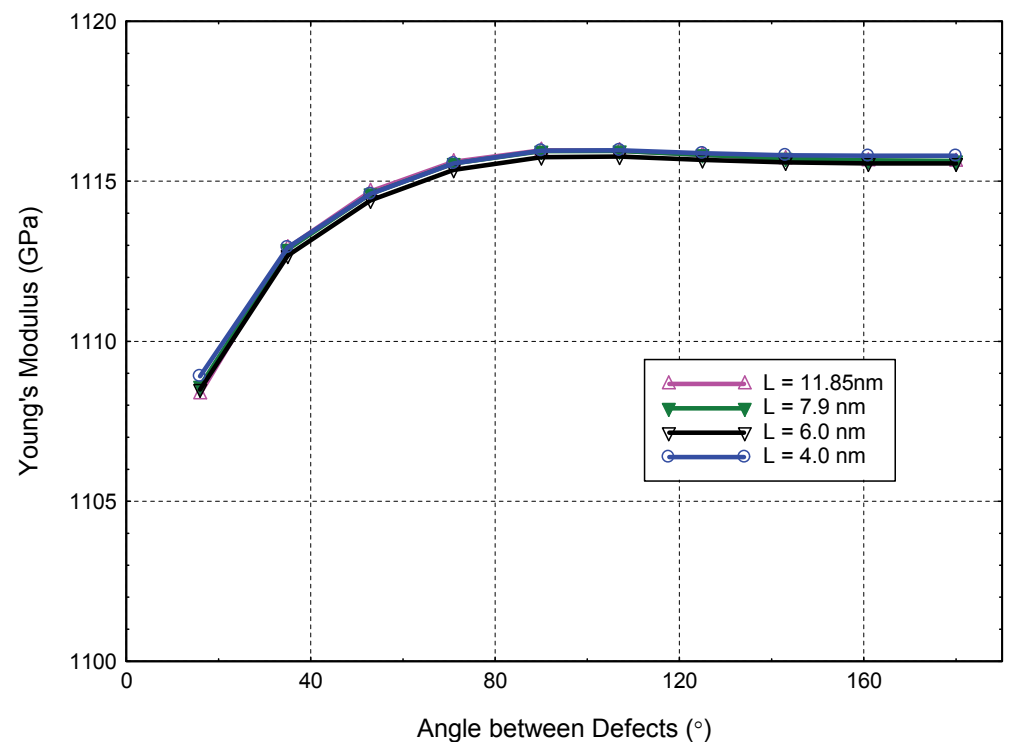

Fig. 13. Young's modulus vs. angle between two defects for $(20,20)$ CNTs with different lengths (Young's modulus calculated using local length of $2 \mathrm{~nm}$ )

In Fig. 13, it can be seen that $E_{\text {local }}$ also exhibits a plateau modulus but is insensitive to the tube length. Results for all tube lengths collapse to a single curve. For angles less than 72 degrees, we see the same drop-off in modulus due to defect interaction. It should be noted that one can use different defective lengths other than $2 \mathrm{~nm}$ to obtain different defective moduli $E_{\text {local }}$. We chose $2 \mathrm{~nm}$ as the size of the affected area based on the study on single defect formation (Xiao, et al., 2009) as the defective length.

The relationship between Young's modulus and defect angle in Figs. 12 and 13 indicates that two defects start to interact when their distance (hoop direction) is smaller than $2 \mathrm{~nm}$ (about 72 degree defect angle). In Fig. 14, we study defect interactions by increasing the number of defects at a given axial location to look at the effects of defect number on the Young's Modulus. In this case we have considered multiple defects that are spaced at uniform angular increments around the circumference (e.g. 4 defects correspond to a separation angle of 90 degrees). Fig. 14 illustrated the difference between our two moduli definitions ( $E_{\text {total }}$ and $\left.E_{\text {local }}\right)$ computed from original tube lengths and local defective length $(2 \mathrm{~nm})$ for the four- and eight-defect cases. The local modulus which is insensitive to overall tube length shows that the modulus drops from the defect-free value as the number of defects increases ( 4 and 8 defects reduce the modulus by 3 and $6 \%$, respectively). For the case of apparent modulus $\left(E_{\text {total }}\right)$ at a given tube length, an increase in the number of defects in the hoop direction reduces the modulus. As the tube length increases, the modulus monontonically increases and approaches the defect-free level. As explained above, this is a consequence of defining strain as change in axial length over initial length. Local deformations around the defect are identical but contribute less to the overall deformation as length increases. One concludes from Fig. 14 that for a given defect pattern, one may take the local modulus (red lines) as the lower bound of the apparent modulus (blue lines) and the defect-free modulus (black line) as the upper bound as the tube length varies. 


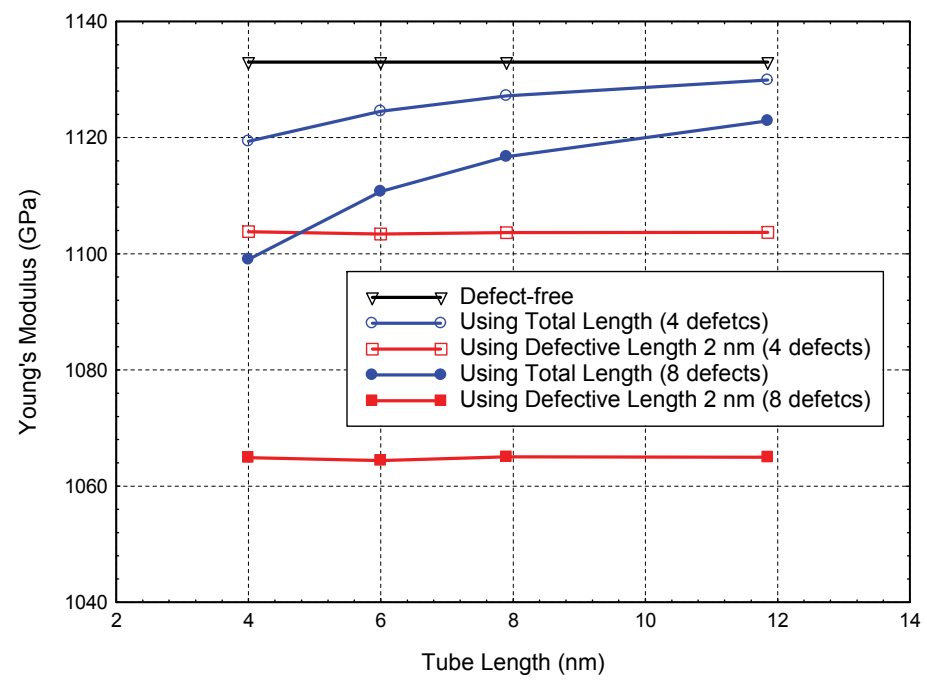

Fig. 14. Young's moduli of $(20,20)$ CNTs with different lengths (4 or 8 defects uniformly distributed along the hoop direction)

The computed moduli of tubes with higher number of defects ranging from 1 to 20 along its hoop direction are presented in Fig. 15. Similar trends are observed where modulus reductions increase with increasing number of defects that are interacting. The local modulus provides a lower bound and the apparent modulus increases with tube length. The results converge to the defect-free result as the number of defects approach zero and the tube length increases. At these higher levels of defects, modulus reductions are quite significant (e.g. local modulus decreases by $30 \%$ for the case of 20 circumferential defects).

One may now generalize the present results to study nanotubes with defects uniformly distributed along both the hoop and axial directions of the tube. Consider each combination of defect number and tube length in Fig. 15 as a unit cell taken from a longer tube with a periodic defect pattern along the tube length. For instance, the calculated Young's modulus of the $6 \mathrm{mn}$ long tube with 8 defects uniformly distributed along its hoop direction, as shown in Fig. 15, can be treated as that of any longer tubes with the 8 hoop defects distributed every $6 \mathrm{~nm}$ along its axial direction. Consequently, one can define defect density as the number of defects per unit surface area within the unit cell of interest. This approach is, based on the assumption that defects exist with equal probability along the entire length of the tube. By following this approach, modulus as a function of defect density is calculated as shown in Fig. 16. Results are now independent of tube length (i.e. local and apparent moduli are equivalent for a given defect density). As the defect density approaches zero, the modulus approaches the defect-free value as expected. Interestingly a linear relationship is observed with modulus decreasing with increasing defect density. A family of curves are presented in Fig. 16 that illustrate the effects of defect interaction for a given defect density. As the separation angle decreases (i.e. interaction increases), the negative slope of the modulus reduction versus defect density increases. The modulus results presented in Fig. 16 are expected to apply to nanotubes of larger diameter than considered in this study $(>2.71 \mathrm{~nm})$ including graphene sheets. The effects of defects on nanotube strength are considered next. 


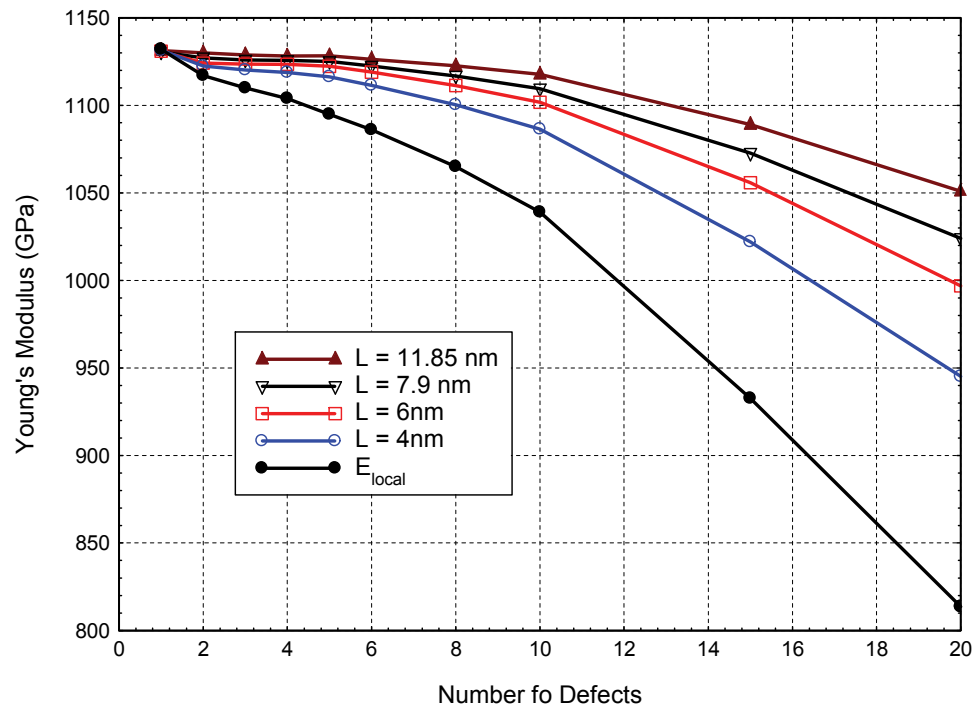

Fig. 15. Young's modulus vs. number of defects for $(20,20)$ CNTs with different lengths

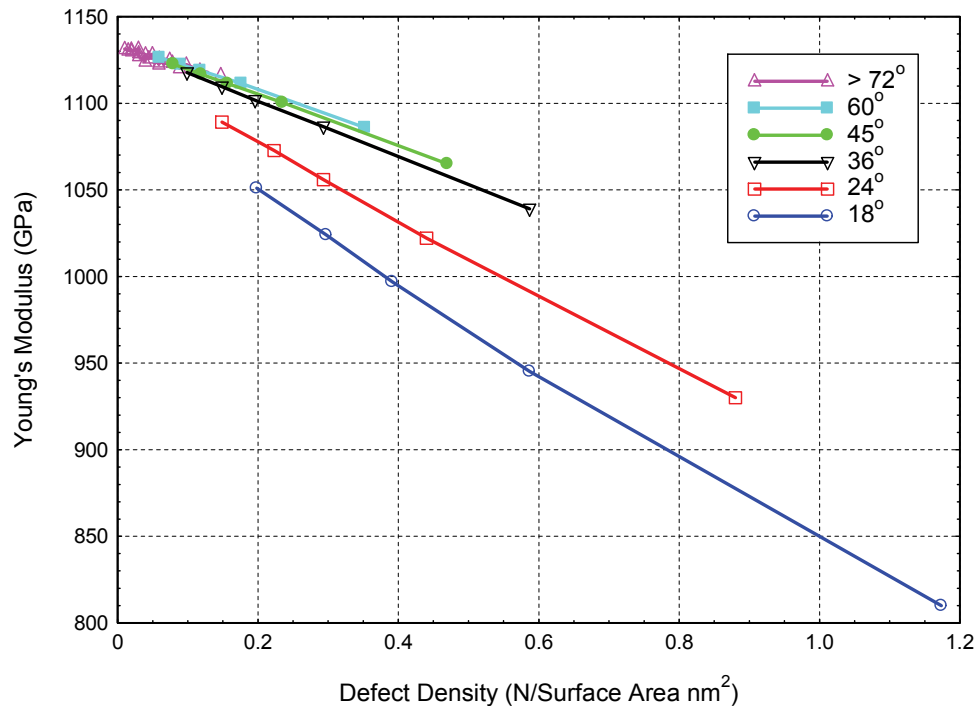

Fig. 16. Young's modulus vs. defect density for a $(20,20) \mathrm{CNT}$

\subsubsection{Effects on ultimate strength}

The effects of isolated defects (i.e. no interactions) on strength are first examined for a $4 \mathrm{~nm}$ long $(10,10)$ nanotube. The ultimate strengths for a defect free tube, a tube with one defect and a tube with two defects along the axial direction at different hoop locations are $124 \mathrm{GPa}$, $84.8 \mathrm{GPa}$, and $84.2 \mathrm{GPa}$ respectively. In contrast to the modulus results discussed above, single defects significantly reduce failure strength (approximately 30\%). Despite having two 
defects along the axial direction, failure strength is effectively the same as a single defective tube, because the determining factor for ultimate strength is the weakest cross-section. These results illustrate that multiple defects that are sufficiently separated such that there are no interactions between them have comparable strength.

To study the effects of multiple interacting defects, let us focus on tubes with defects distributed around their hoop direction. Similar to the Young's modulus study, simulations were conducting using $(10,10),(20,20),(17,0)$, and $(35,0)$ nanotubes with different lengths of 4 , and 6 nanometers. It should be noted that a $(10,10)$ tube has about the same diameter as a $(17,0)$ tube, and the same is true for $(20,20)$ and $(35,0)$. In each simulation, the tube was loaded quasi-statically in tension under prescribed displacement until failure. For failure of a bond to occur, the individual strain of the bond had to reach $18.5 \%$. This value is consistent with published data as an inflection point for a C-C bond. The simulations predict that once a single initial bond has broken, the stress would drop abruptly under displacement controlled loading. The basic nanotube structure will still be intact and have some post-failure properties. In our simulations, the ultimate strength is defined as the maximum stress the tube reaches. Also, the strain at failure is the strain that corresponds to this ultimate stress.

In Fig. 17, we consider the armchair cases with two defects at the same axial location as a function of separation angle. At angles greater than approximately 70 degrees a strength plateau is established equal to the ultimate strength of a single defect mentioned above. When the angle drops below 70 degrees $(0.83 \mathrm{~nm}$ distance for a $(20,20) \mathrm{CNT}$, which is about the same size of the defective area), the defects begin to interact and a dramatic reduction in strength is predicted. For the case of 18 degree separation angle, the strength has dropped $58 \%$ of the defect-free ultimate strength. Results from the $(10,10)$ CNT simulation follow the same trend. In contrast to the modulus results, it should be noted that the computed strengths are not sensitive to tube length.

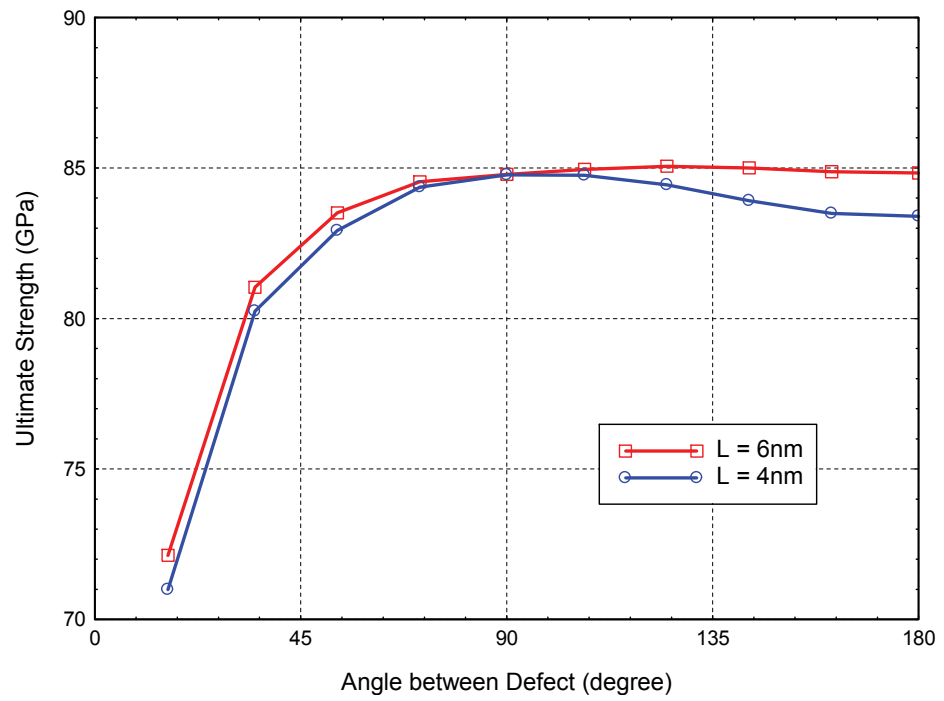

Fig. 17. Ultimate strength vs. angle between two defects for $(20,20)$ CNTs with different lengths 


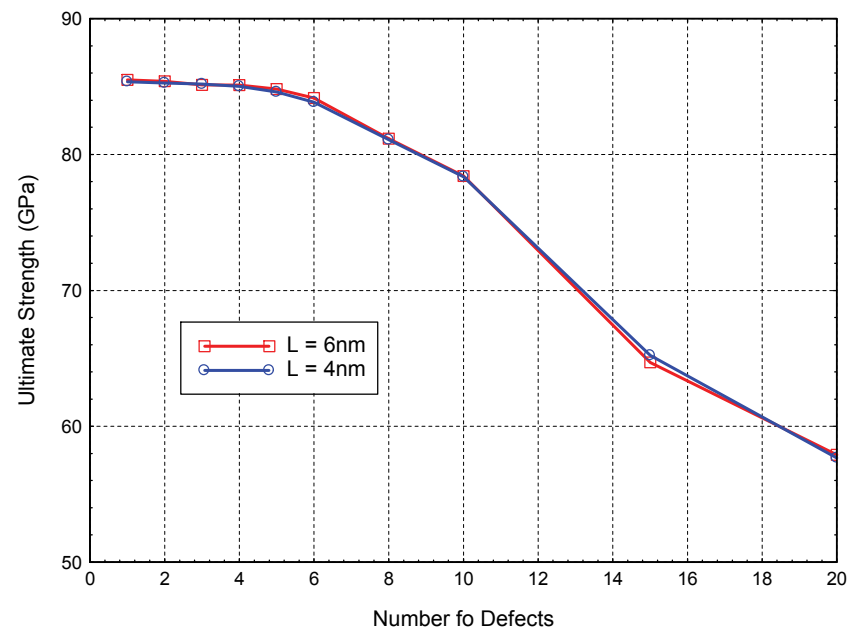

Fig. 18. Ultimate strength vs. Number of defects for $(20,20)$ CNTs with different lengths

The effect of multiple defects at the same axial location on the ultimate strength is considered next. In the armchair configuration, increasing the amount of defects present around the circumference decreases the ultimate strength as shown in Fig. 18 from the plateau strength corresponding to a single defect. At approximately 4-6 defects, the separation angle drops below 70 degrees and significant interactions between multiple defects develop. In the case of multiple defects, the strength drops to the same level as the results for 2 defects separated by the same angle. For example, 20 defects have a separation angle of 18 degrees and one observes the same strength reduction as shown in Fig. 18 (i.e. $58 \%$ of the defect-free tube). One concludes that the strength of a tube with multiple defects is determined by the degree of interaction indicated by the separation distance.

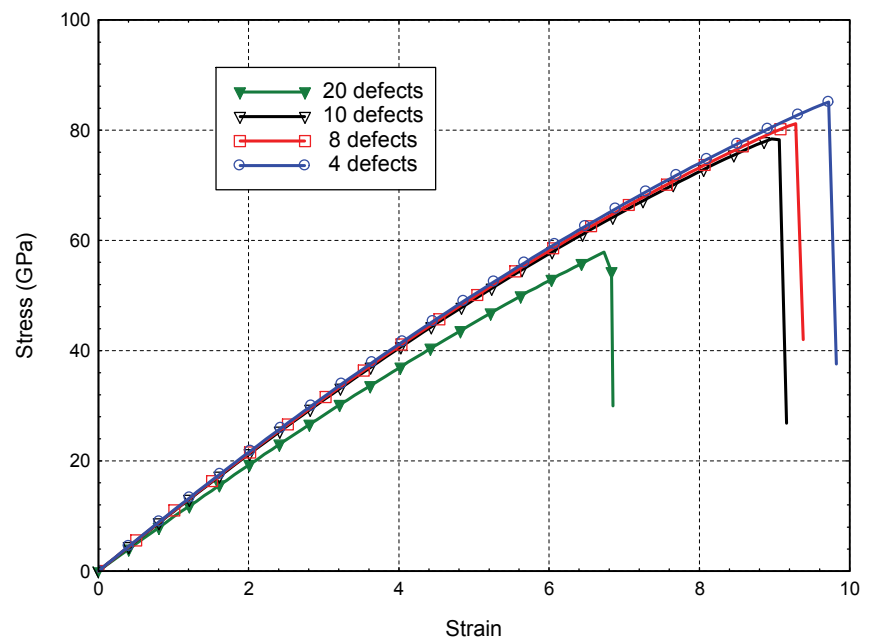

Fig. 19. Stress-strain curves of a $6 \mathrm{~nm}(20,20) \mathrm{CNT}$ with multiple defects equally spaced around the circumference at the middle section 
Fig. 19 gives the computed stress-strain relationships of a $6 \mathrm{~nm}(20,20)$ tube with different number of defects spaced uniformly around the circumference at the same axial location (about the middle section of the tube). Based on the discussion above, the initial modulus decreases as the number density of defects increases. In addition, the strength and strain to failure decreases as the separation angle between defects decreases. In this figure, the 20 defect curve has both the lowest modulus and lowest ultimate strength and strain to failure. The failure patterns of the $6 \mathrm{~nm}(20,20)$ tube with 4 and 8 defects equally spaced around the circumference direction under tension loading are shown in Fig. 20. It can be seen that the failure of the tube with 8 defects (Fig. 20b) is extremely localized with no diagonal crack propagation as seen in the tube with less hoop defects (see Fig. 11 and Fig. 20a).

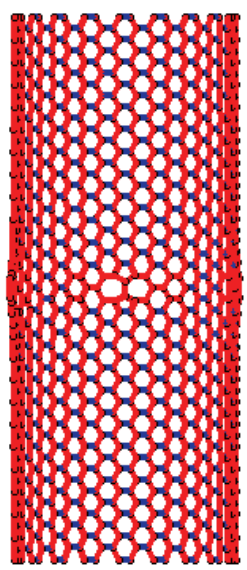

(a) With 4 defects

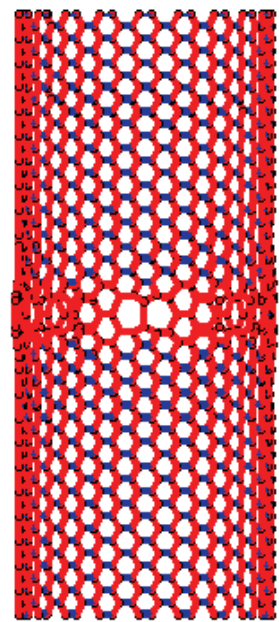

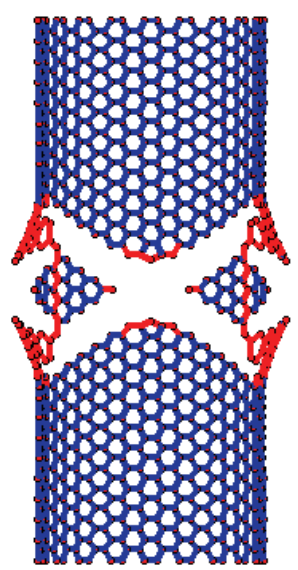

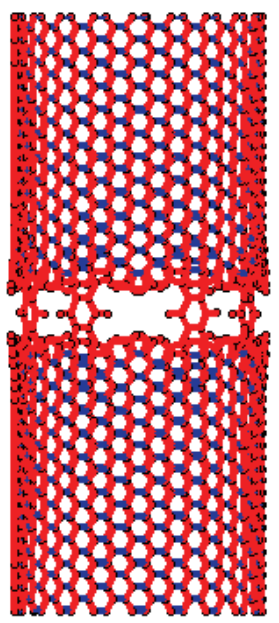

(b) With 8 defects

Fig. 20. Failure patterns of a $6 \mathrm{~nm}(20,20)$ CNT with multiple defects equally spaced around the circumference at the middle section 


\section{Conclusions}

By incorporating the modified Morse potential function into a novel atomistic finite bond element molecular structural mechanics model, the mechanical responses of graphene sheet and single-walled nanotubes under tension conditions are investigated. The finite bond element has 8 degrees of freedom (DOF) which is used to simulate the interatomic connections in CNTs. Compared to other similar finite element approaches (12DOFs) the computational cost of the present method is reduced. The coefficients in the finite bond element model are taken from the analytical molecular mechanics model (Xiao et al., 2005). The present approach is capable of predicting Young's moduli, Poisson's ratios, and stressstrain relationships of graphene sheets and nanotubes with or without a SW defect. An interaction mechanics approach is introduced to model the formation of a 5-7-7-5 SW defect in CNTs which reasonably captures the physical phenomena in terms of reconfiguration, local deformation and formation energy. Consequently, effects of the SW defect on the Young's moduli, fracture and progressive failure of defective CNTs have been investigated. Using the present approach, it is feasible to model multiple defects and their interaction in both SWCNT and MWCNT since the present approach is much simpler and computationally efficient than the classical molecular dynamics model.

A methodology to create multiple defects in a CNT at given locations has been implemented into our MATLAB code. Consequently, the effect and interaction of multiple defects on a SWCNT were studied. The resulting simulations were able to predict the Young's modulus, ultimate strength, and strain at failure. The influence of single and multiple defects on mechanical properties were studied. In the case of Young's modulus, increasing the number of defects along the hoop direction can change its properties dramatically, particularly when the defect distance is smaller than $2 \mathrm{~nm}$ (defect interaction). It is also found that moduli are sensitive to the tube lengths when the total tube length is used to compute the strain. This finding has major implications for comparing experimental data from tests on different tube lengths. A local defective length $(2 \mathrm{~nm})$ is introduced to separate the overall deformation into two different regions: defective and defect-free. By doing so, a size independent modulus has been obtained for the defective region, which can be treated as the minimum modulus for a given defective tube of any length larger than $2 \mathrm{~nm}$.

In addition, results were generalized to consider defect density (number of defects per unit surface area). Stiffness reductions were found to be linearly related to defect density (number of defects per unit surface area) and to become independent of tube length. It was found that further reductions occur when the defects interact with each other (typically when the distance between defects is smaller than $2 \mathrm{~nm}$ ). The effects of single and multiple defects on ultimate strength were considered. Significant strength reductions are predicted to occur by the presence of single defects. Further strength reductions are predicted for cases where adjacent defects are interacting. In this case the ultimate strength is governed more by the separation distance than the defect density. Finally the overall stress-strain response has been predicted as a function of number of defects. Results including the effects of defects more closely match experimental data reported in the literature. It should be noted that this present model has a more simple approach than quantum or the classical molecular dynamics model and is less computationally expensive.

\section{References}

Abell GC. (1985). Empirical chemical pseudopotential theory of molecular and metallic bonding. Physical Review B, Vol. 31, pp.6184-6196. 
Belytschko T.; Xiao SP.; Schatz GC.; \& Ruoff RS. (2002). Atomistic simulations of nanotube fracture. Physical Review B, Vol. 65, pp.235430.

Brenner DW. (1990). Empirical potential for hydrocarbons for use in simulating the chemical vapor-deposition of diamond films. Physical Review B, Vol. 42, pp.9458-9471.

Cadek M.; Coleman JN.; Barron V, et al., (2002). Morphological and mechanical properties of carbon-nanotube-reinforced semicrystalline and amorphous polymer composites. Appl. Phys. Lett.; Vol. 81, pp.5123-5125

Chang T.; \& Gao H. (2003). Size-dependent elastic properties of a single-walled carbon nanotube via a molecular mechanics modelnanotubes. J. the Mech. Phys. Solids, Vol.51, pp.1059 -1074.

Che J, Cagin T, \& Goddard WA, (2000). Thermal conductivity of carbon nanotubes. Nanotechnology, Vol. 11, pp. 65-69.

Cornell WD.; Cieplak P, Bayly CI, et al., (1995). A second generation force field for the simulation of proteins, nucleic acids, and organic molecules. American Chem. Soc. Vol.117, pp.5179-5197.

Dalton AB.; Collins S.; Munoz E.; et al., (2003). Super-tough carbon-nanotube fibres - These extraordinary composite fibres can be woven into electronic textiles. Nature (London). Vol. 423, pp.703-703.

Dresselhaus MS.; Dresselhaus G.; \& Eklund PC, (1996). Science of fullerenes and carbon nanotubes, Academic Press, San Diego.

Dumitrica T.; Belytschko T.; Yakobson BI, (2003). Bond-breaking bifurcation states in carbon nanotube fracture. J. Chem. Phys. Vol.118(21), pp.9485-9488.

Kanchi MB. (1993). Matrix Methods of Structural Analysis, John Willey \& Sons,.

Huhtala M, Krasheninnikov AV, Aittoniemi J, et al., (2004). Improved mechanical load transfer between shells of multiwalled carbon nanotubes. Physical Review B, vol.70(4), pp.045404

Huxtable ST, Cahill DG, Shenogin S, et al., (2003). Interfacial heat flow in carbon nanotube suspensions. Nat. Mater. Vol. 2, pp.731-734.

Iijima S. (1991). Helical microtubes of graphitic carbon. Nature, Vol. 354, pp. 56-58.

Jiang H, Feng XQ, Huang Y, et al., (2004). The effect of nanotube radius on the constitutive model for carbon nanotubes. Comput. Methods Appl. Mech. Engrg. Vol.193, pp.3419-3429.

Jiang H, Zhang P, Liu B, Huang Y, et al., (2203). The effect of nanotube radius on the constitutive model for carbon nanotubes. Computational Material Science. Vol. 28, pp.429-442.

Jorgensen WL. \& Severance DL, (1990). Aromatic aromatic interactions - free-energy profiles for the benzene dimer in water, chloroform, and liquid benzene. American Chem. Soc. Vol.112, pp.4768-4774.

Li CY. \& Chou TW, (2003). A structural mechanics approach for the analysis of carbon nanotubes. Int. J. Solids Struct. Vol.40, pp.2487-2499.

Lu Q \& Bhattacharya B, (2005). Fracture resistance of zigzag single walled carbon nanotubes. Nanotechnology. Vol.16, pp.555-566. 
Lu Q \& Bhattacharya B, (2005). The role of atomistic simulations in probing the small-scale aspects of fracture - a case study on a single-walled carbon nanotube. Eng. Fract. Mech. Vol.72, pp.2037-2071.

Nardelli MB, Yakobson BI, \& Bernholc J, (1998). Mechanism of strain release in carbon nanotubes. Physical Review B, Vol.57, pp.R4277

Pan C, Yang WS, \& Yang J, (2000). Formation energies of topological defects in carbon nanotubes. Physical Review B, Vol.62, pp.12652.

Sears A, Batra RC, (2004). Macroscopic properties of carbon nanotubes from molecularmechanics simulations. Physical Review B, Vol. 69, pp.235406.

Stone AJ, Wales DJ, (1986). Theoretical studies of icosahedral c60 and some related species. Chem. Phys. Lett. Vol.128, pp.501-503.

Tersoff J, (1988). Empirical interatomic potential for carbon, with applications to amorphouscarbon.Phys. Rev. Let. Vol.61, pp.2872-2879.

Thostenson ET, Ren Z, Chou TW. (2001). Advances in the science and technology of carbon nanotubes and their composites: a review. Composites Sci. Tech.Vol.61, pp.1899_ 1912.

Tserpes KI, Papanikos P, (2007). The effect of Stone-Wales defect on the tensile behavior and fracture of single-walled carbon nanotubes. Comp. Struct. Vol. 79, pp.581-589.

Wagner HD, Lourie O, Feldmann Y, \& Tenne R, (1998). Stress-induced fragmentation of multiwall carbon nanotubes in a polymer matrix. Appl Phys Lett. Vol. ;72, pp.188190.

Wong EW, Sheehan PE, \& Lieber CM, (1997). Nanobeam mechanics: elasticity, strength, and toughness of nanorods and nanotubes. Science. Vol. 277, pp.1971-1975.

Xia Y, Zhao M, Ma Y, et al., (2002). Tensile strength of single-walled carbon nanotubes with defects under hydrostatic pressure. Physical Review B, Vol. 65, pp.155415.

Xiao JR, Gama BA, \& Gillespie JW, (2005). An analytical molecular structural mechanics model for the mechanical properties of carbon nanotubes. Int. J. Solids Struct.;vol. 42, pp.3075-3092.

Xiao JR, Lopatnikov S, Gama BA, \& Gillespie JW, (2006) Nanomechanics on the deformation of single- and multi-walled carbon nanotubes under radial pressure. Materials Science and Engineering A. Vol. 416, pp.192-204.

Xiao JR \& Gillespie JW. ( 2006a). Nonlinear deformation and progressive failure of multiwalled carbon nanotubes under internal radial pressure Physical Review B, Vol. 74, pp.155404

Xiao JR \& Gillespie JW. (2006b). Nanomechanics of single-walled carbon nanotubes as composite reinforcement. Polymer Eng. Sci.; Vol. 46, pp.1051-1059.

Xiao SP \& Hou WY. (2006). Studies of nanotube-based resonant oscillators through multiscale modeling and simulation. Physical Review B, Vol. 73, pp.115406.

Yakobson BI \& Avouris P. (2001). In Carbon Nanotubes. Topics in Applied Physics, Dresselhaus, M.S., Dresselhaus, G., Avouris, P. (Eds.), Springer Verlag, Berlin-Heidelberg, Germany. V. 80, p.287.

Ye LH, Liu BG, \& Wang DS, (2001). Ab initio molecular dynamics study on small carbon nanotubes. Chinese Phys. Lett., Vol. 18, pp.1496-1499. 
Yu MF, Files BS, Arepalli S, \& Ruoff RS, (2000). Strength and breaking mechanism of multiwalled carbon nanotubes under tensile load. Phys. Rev. Lett.; vol. 84, pp.55525555.

Zhang P, Huang Y, Geubelle PH, et al., (2002). The elastic modulus of single-wall carbon nanotubes: a continuum analysis incorporating interatomic potentials. International Journal of Solids Structures, Vol. 39, pp.3893-3906.

Zhang S, Mielke SL, Khare R, et al., (2005). Mechanics of defects in carbon nanotubes: Atomistic and multiscale simulations. Physical Review B, Vol. 71, pp.115403 


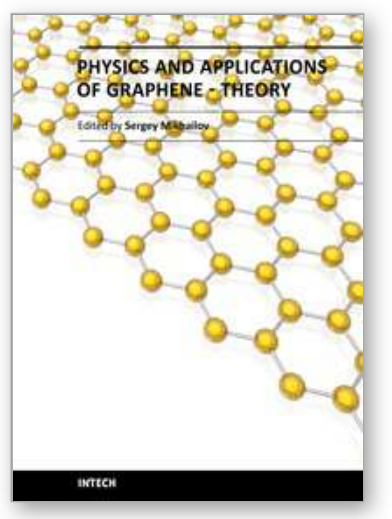

\section{Physics and Applications of Graphene - Theory}

Edited by Dr. Sergey Mikhailov

ISBN 978-953-307-152-7

Hard cover, 534 pages

Publisher InTech

Published online 22, March, 2011

Published in print edition March, 2011

The Stone Age, the Bronze Age, the Iron Age... Every global epoch in the history of the mankind is characterized by materials used in it. In 2004 a new era in material science was opened: the era of graphene or, more generally, of two-dimensional materials. Graphene is the strongest and the most stretchable known material, it has the record thermal conductivity and the very high mobility of charge carriers. It demonstrates many interesting fundamental physical effects and promises a lot of applications, among which are conductive ink, terahertz transistors, ultrafast photodetectors and bendable touch screens. In 2010 Andre Geim and Konstantin Novoselov were awarded the Nobel Prize in Physics "for groundbreaking experiments regarding the two-dimensional material graphene". The two volumes Physics and Applications of Graphene - Experiments and Physics and Applications of Graphene - Theory contain a collection of research articles reporting on different aspects of experimental and theoretical studies of this new material.

\section{How to reference}

In order to correctly reference this scholarly work, feel free to copy and paste the following:

J. R. Xiao and J. W. Gillespie Jr. (2011). Fracture Behaviors of Graphene Sheets and Carbon Nanotubes, Physics and Applications of Graphene - Theory, Dr. Sergey Mikhailov (Ed.), ISBN: 978-953-307-152-7, InTech, Available from: http://www.intechopen.com/books/physics-and-applications-of-graphene-theory/fracturebehaviors-of-graphene-sheets-and-carbon-nanotubes

\section{INTECH}

open science | open minds

\section{InTech Europe}

University Campus STeP Ri

Slavka Krautzeka 83/A

51000 Rijeka, Croatia

Phone: +385 (51) 770447

Fax: +385 (51) 686166

www.intechopen.com

\section{InTech China}

Unit 405, Office Block, Hotel Equatorial Shanghai

No.65, Yan An Road (West), Shanghai, 200040, China 中国上海市延安西路65号上海国际贵都大饭店办公楼 405 单元

Phone: +86-21-62489820

Fax: $+86-21-62489821$ 
(C) 2011 The Author(s). Licensee IntechOpen. This chapter is distributed under the terms of the Creative Commons Attribution-NonCommercialShareAlike-3.0 License, which permits use, distribution and reproduction for non-commercial purposes, provided the original is properly cited and derivative works building on this content are distributed under the same license. 